\title{
Usefulness of a Hanging Position With Internal Rotation of Shoulder in Ultrasonography- Guided Intra-articular Steroid Injection for Adhesive Capsulitis
}

\author{
Chang Han Lee, $\mathrm{MD}^{1}$, Hyung Seok Nam, $\mathrm{MD}^{2,3}$, Shi-Uk Lee, MD, $\mathrm{PhD}^{3}$ \\ ${ }^{1}$ Department of Rehabilitation Medicine, Gyeongsang National University Hospital, \\ Gyeongsang National University Graduate School of Medicine, Jinju; \\ ${ }^{2}$ Department of Biomedical Engineering, Seoul National University College of Medicine, Seoul; \\ ${ }^{3}$ Department of Rehabilitation Medicine, Seoul National University Boramae Medical Center, Seoul, Korea
}

Objective To evaluate the feasibility of a new position (internal rotation in hanging) in ultrasonography, we compared the length of the glenohumeral joint space and the effectiveness of steroid injection with the hanging position and with the commonly used abdomen or cross position.

Methods A prospective, randomized controlled trial was performed in 42 patients with adhesive capsulitis of shoulder. We used three arm positions for the posterior approach as follows: the patient's palm on thigh, other hand on abdomen (abdomen position); hand on patient's opposite shoulder (cross position); arm in hanging position with internal rotation of shoulder (hanging position). The order of shoulder position was randomized and blinded. Real-time ultrasonography-guided intra-articular steroid injection was performed by posterior approach at the first position in each patient. The Brief Pain Inventory (BPI), the Shoulder Pain and Disability Index (SPADI), and range of motion (ROM) were measured before steroid injection and 2 weeks after injection.

Results The lengths of the joint space were $2.88 \pm 0.75,2.93 \pm 0.89$, and $2.82 \pm 0.79 \mathrm{~mm}$ in abdomen, cross, and hanging position respectively, with no significant difference among the three positions $(\mathrm{p}=0.429)$. Treatment efficacy was significantly improved in ROM, total BPI, and SPADI in all three positions $(\mathrm{p}<0.001)$. The changes in ROM for shoulder abduction were $23.6^{\circ} \pm 19.7^{\circ}, 22.2^{\circ} \pm 20.9^{\circ}$, and $10.0^{\circ} \pm 7.8^{\circ}$ in abdomen, cross, and hanging position, respectively. Changes in total BPI scores were 25.1 $\pm 15.7,23.6 . \pm 18.0,11.6 \pm 6.1$, and changes in total SPADI score were $35.0 \pm 14.2,30.9 \pm 28.9$, and $16.5 \pm 10.3$ in abdomen, cross, and hanging position, respectively. There were no significant difference among the three positions for all parameters $(\mathrm{p}=0.194, \mathrm{p}=0.121$, and $\mathrm{p}=0.108$,

Received February 22, 2016; Accepted April 6, 2016

Corresponding author: Shi-Uk Lee

Department of Rehabilitation Medicine, Seoul National University Boramae Medical Center, 20 Boramae-ro 5-gil, Dongjak-gu, Seoul 07061, Korea. Tel: +82-2-870-2671, Fax: +82-2-870-3866, E-mail: ShiUk.Lee@gmail.com

ORCID: Chang Han Lee (http://orcid.org/0000-0001-8351-5226); Hyung Seok Nam (http://orcid.org/0000-0002-2210-7170); Shi-Uk Lee (http://orcid. org/0000-0003-0850-5217).

() This is an open-access article distributed under the terms of the Creative Commons Attribution Non-Commercial License (http://creativecommons.org/ licenses/by-nc/4.0) which permits unrestricted noncommercial use, distribution, and reproduction in any medium, provided the original work is properly cited. Copyright $\odot 2016$ by Korean Academy of Rehabilitation Medicine 
respectively.

Conclusion For patients with adhesive capsulitis who cannot achieve or maintain abdomen or cross position, scanning and injection with the shoulder in internal rotation with hanging position may be a useful alternative.

Keywords Adhesive capsulitis, Posture, Intra-articular injections, Ultrasonography

\section{INTRODUCTION}

Adhesive capsulitis, one of the most common causes of shoulder pain, is a clinical syndrome characterized by spontaneous onset of shoulder pain with restriction of both active and passive motion $[1,2]$. Adhesive capsulitis is mostly a self-limiting disorder, although it takes several months or even years to heal [3]. At present there is no consensus on proper treatment for adhesive capsulitis, although several methods of treatment, such as physical therapy, pharmaceutical intervention, intra-articular steroid injection, and exercise therapy are in clinical use [4]. Of these methods, intra-articular steroid injection is most commonly used to treat adhesive capsulitis of the shoulder [5,6], and intra-articular steroid injection under real-time ultrasonographic control has been reported recently. Two approaches are used in the intra-articular steroid injection under ultrasonography (IA-USG), anterior and posterior [7-12]. The more commonly used approach is the posterior approach which has been proven to be an effective and cost-effective option for the treatment of adhesive capsulitis of the shoulder $[8,9,12]$.

According to literature reports, the usual arm positions for the posterior approach is to place the patient's palm on the thigh and the hand of the affected arm on the abdomen (abdomen position) [8,9], or the hand on the patient's opposite shoulder (cross position) [10] (Fig. 1). The main purpose of various hand positions is to increase the size of the glenohumeral (GH) joint space so that the intra-articular injection will be easier to accomplish. Anatomically, the posterior GH joint space increases as the degree of internal rotation of the humerus increases. With the abdomen position, the degree of internal rotation is limited by the patient's own abdomen. Using the cross position, more internal rotation of the humerus may be achieved. However, patients sometimes have difficulties in placing their hand on the opposite shoulder because of shoulder pain. This pain may result from the limited range of motion (ROM) of the shoulder, not only in internal rotation, but also in flexion and adduction in adhesive capsulitis patients.

With the hand in the hanging position as shown in Fig. 1, the degree of internal rotation of the humerus is greater, and the patient is more comfortable because the hanging position does not require additional shoulder motion. Therefore, the hanging position provides benefits not only for physicians who perform the injection by increasing the GH joint space, but also for patients because it is
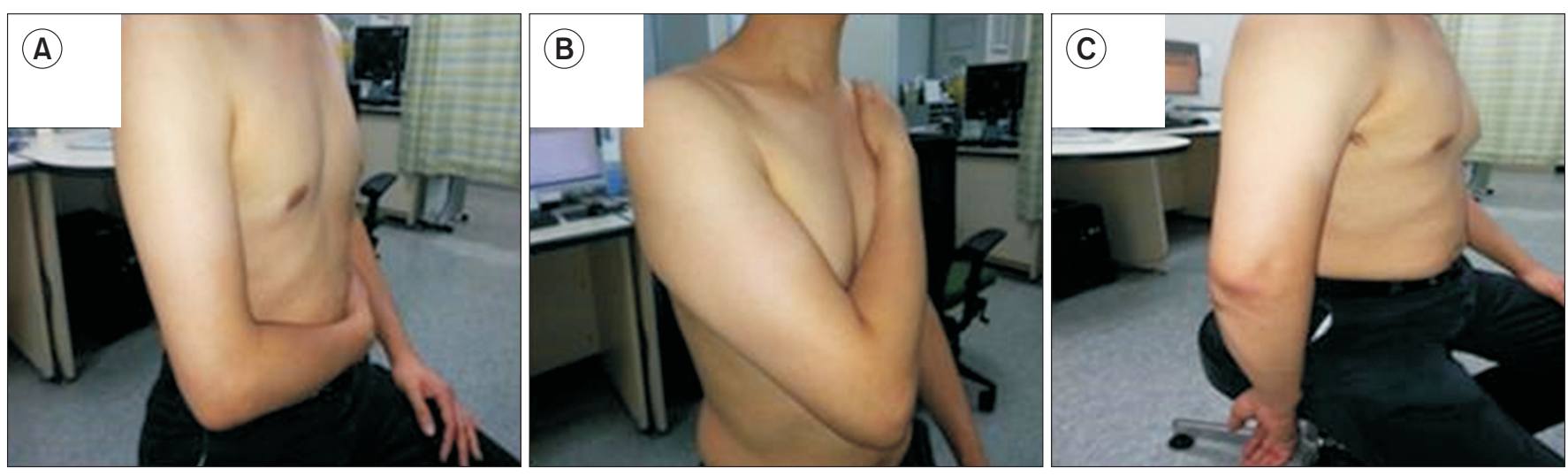

Fig. 1. Three positions of the shoulder during intra-articular injection are shown. (A) Hands on abdomen (abdomen position), (B) hands on opposite shoulder (cross position), and (C) internal rotation with hanging (hanging position). 
more comfortable.

To our knowledge, the feasibility of the hanging position has not been reported. The purpose of this study is to evaluate the feasibility of the hanging position by comparing its effect on the length of the GH joint space measured by ultrasonography and its effectiveness compared to the two commonly used positions (abdomen and cross positions).

\section{MATERIALS AND METHODS}

\section{Subjects}

A prospective, randomized, and controlled trial study was performed using 42 patients, with informed consent, who visited our rehabilitation outpatient department from June 2013 to August 2013. They were selected based on a medical interview, physical examination, radiological examination, and musculoskeletal ultrasonography in order to diagnose adhesive capsulitis. Patients with stage II or greater shoulder adhesive capsulitis were included [13]. The criteria for selection were as follows: (1) pain at rest; (2) pain above the elbow; (3) disturbed sleep on the painful side at night; (4) hard end-feel on external rotation; (5) limited ROM in flexion, abduction, and/or external rotation of more than $30^{\circ}$ compared to the normal side $[14,15]$. Exclusion criteria were the following: complete rotator cuff tear as seen with ultrasonography, past traumatic shoulder injury requiring hospitalization in the prior 6 months, severe mental illness, age of less than 18 years or more than 70 , degenerative arthritis in the shoulder (detected by radiological studies), systemic inflammatory disease, current use of anti-coagulant medications, and current infectious disease or suspected infection $[16,17]$. This study was approved by the Institutional Review Board of Gyeongsang National University Hospital (IRB No. 20130430/26-2013-37/052).

\section{Methods}

Ultrasonography scans were performed by two clinicians with expertise in musculoskeletal ultrasonography. Images were acquired with LOGIQ P5 (GE Healthcare, Seoul, Korea) and a 4.5-12.0 MHz probe. The patient's shoulder was scanned with the shoulder in each of the three different positions described previously-abdomen, cross, and hanging positions (Fig. 1). The order of patients' shoulder position was randomized by using a table of random numbers. Each patient was randomly assigned into one of six groups: $\mathrm{ACH}$ (A, abdomen position; C, cross position; $\mathrm{H}$, hanging position; $\mathrm{A}$ as 1st, $\mathrm{C}$ as 2nd, and $\mathrm{H}$ as 3rd position), AHC, $\mathrm{CAH}, \mathrm{CHA}, \mathrm{HAC}$ and HCA. Images were saved in the digital imaging and communication in medicine files of the picture archiving communication system (M-View 5.4; Marotech, Seoul, Korea). After obtaining the three images for each patient, intra-articular steroid injection under real-time ultrasonography control was performed with the shoulder of each patient in the first position. To blind the measurer, the annotation of each image was numbered from 1 to 3 according to the order of shoulder position. This avoids an observational bias that might occur in the case of preferred positions of clinicians.

The space between the lateral edge of the labrum and hyaline cartilage of the humerus is the space where the needle is inserted during the procedure. The length of this space was evaluated by two separate blinded clinicians. The length was determined by measuring the perpendicular line between the starting point of the free

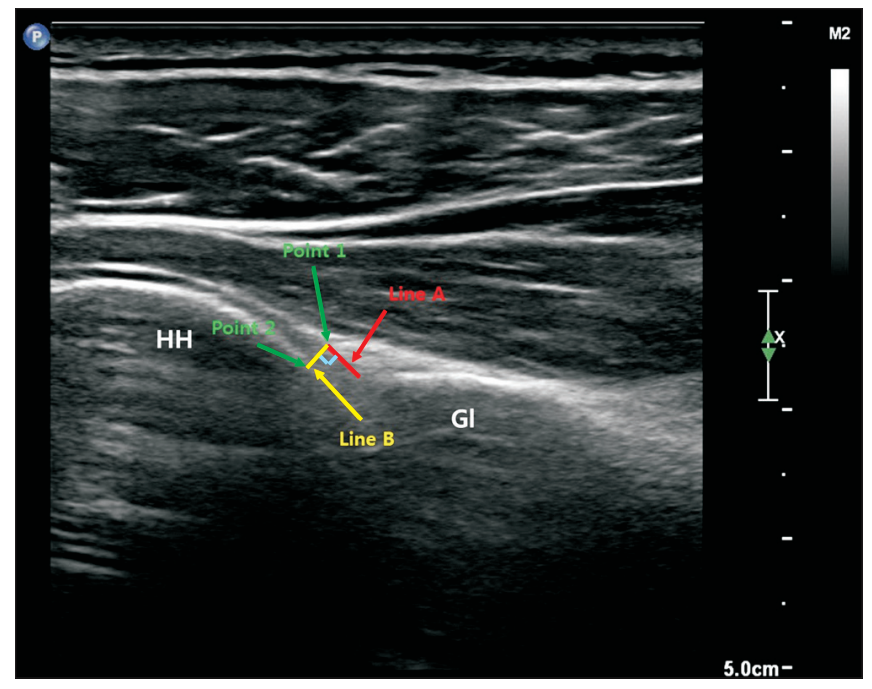

Fig. 2. Method used to measure the length of glenohumeral joint in ultrasonography. A line was drawn from the lateral edge of the labrum (point 1) along the inferior margin of labrum (line A, red line). From the lateral edge of the labrum, a line (line B, yellow line) was drawn perpendicular to the line A. The distance from the lateral edge of the labrum (point 1 ) to the corresponding cortical echo of the humerus (point 2) on the line B was defined as the length of glenohumeral joint. $\mathrm{HH}$, humeral head; Gl, bony glenoid. 
margin of the labrum and the hyaline cartilage of the humerus using the detailed method shown in Fig. 2. Each evaluator made three measurements and an average value was used for comparison.

\section{Efficacy measurements}

Efficacy measurements were performed before steroid injection and 2 weeks after the injection. The degree of shoulder pain was assessed by the Brief Pain Inventory (BPI) [18]. The BPI is composed of a pain severity score and a pain interference score. The BPI pain severity score includes four items that are scored with numeric rating scales from the patient who rate their pain intensity on a 0 to 10 point scale. Each scale is presented as a row of equidistant numbers where 0 is defined as 'no pain' and 10 as 'worst possible pain. The patient's functional status was measured according to seven items using the BPI pain interference score. The BPI pain interference score is scaled from 0 representing 'no interference' to 10 representing 'interference completely' The lower the score, the less shoulder pain and interference. To assess shoulder pain and disability, the Shoulder Pain and Disability Index (SPADI) [19] was used before and after the intra-articular injection of steroid. SPADI consists of two subscales, which are pain and disability. Pain and disability subscales contain 5 and 8 questions, respectively. The total SPADI score is calculated by averaging the subscales. ROM in flexion, abduction, external rotation, internal rotation, external scratch, and internal scratch was assessed for passive movement. The Apley scratch test is one test to assess shoulder flexibility as external and internal scratch [20]. During testing, one shoulder is abducted and externally rotated until the hand touches the upper back, while the contralateral side is adducted and internally rotated to touch the lower back. Measurements are taken between the two index fingers. The positions of the arms are reversed and measurements are repeated.

\section{Statistics}

Comparisons of the difference in the GH joint space in the three arm positions were performed by a repeated measurement of one-way analysis of variance (ANOVA). Bonferroni correction was completed as the post-hoc test. We performed Wilcoxon signed-rank test to compare the shoulder ROM, BPI, and SPADI between pre-injection and 2 weeks after injection. Additionally the Krus-
kal-Wallis test was performed to compare differences of treatment efficacy according to arm position through the shoulder ROM, BPI, and SPADI after injection. Statistical analyses were carried out using PASW ver. 18 (SPSS Inc., Chicago, IL, USA). The level of statistical significance was set at $\mathrm{p}$-value less than 0.05 .

\section{RESULTS}

\section{General characteristics of the subjects}

The subjects were 25 females and 17 males with a mean age of 57.6 years. The average durations and involved side of shoulder pain are summarized in Table 1. There were no significant differences between the position groups in sex, age, duration of symptom, and laterality of involved shoulder. Among 42 patients, 16 patients did not visit our clinic after initial injection. In the 26 patients, 7 patients belonged to abdomen position, 9 patients to cross position, and 10 patients hanging position, respectively.

\section{Comparisons of the efficacy measurements}

In the 26 patients, 2 weeks after injection of steroid, significant improvement in shoulder ROM was noted in all of three arm positions. The total BPI and SPADI were also significantly improved 2 weeks after injection in all three arm positions. Mean scores of total BPI were decreased from $45.4 \pm 21.7$ to $23.7 \pm 21$.2. In case of total SPADI, mean scores were decreased from $61.2 \pm 24.2$ to $34.7 \pm 27.2$ (Table 2).

Table 1. Demographic characteristics of the subjects

\begin{tabular}{|cc|}
\hline Characteristic & Value \\
\hline Sex & $17(40.5)$ \\
\hline Male & $25(59.5)$ \\
\hline Female & $57.55 \pm 10.10$ \\
\hline Age (yr) & $6.4 \pm 8.4$ \\
\hline Duration (mo) & \\
\hline Involved shoulder & $31(73.8)$ \\
\hline Right & $11(26.2)$ \\
\hline Left & 7 \\
\hline Shoulder position & 9 \\
\hline Abdomen & 10 \\
\hline Cross & \\
\hline Hanging & \\
\hline
\end{tabular}

Values are presented as number (\%) or mean \pm standard deviation. 
Chang Han Lee, et al.

Table 2. Comparison of outcome measurements pre-injection and 2 weeks after injection

\begin{tabular}{lccc}
\hline & Pre-injection & 2 weeks after injection & p-value \\
\hline Abduction $\left(^{\circ}\right)$ & $111.4 \pm 30.9$ & $129.2 \pm 32.8$ & $<0.001^{*}$ \\
\hline Flexion $\left(^{\circ}\right)$ & $140.6 \pm 21.8$ & $153.5 \pm 20.3$ & $<0.001^{*}$ \\
\hline External rotation $\left(^{\circ}\right)$ & $57.7 \pm 23.3$ & $69.0 \pm 16.8$ & $<0.001^{*}$ \\
Internal rotation $\left(^{\circ}\right)$ & $47.8 \pm 22.9$ & $62.1 \pm 19.7$ & $<0.001^{*}$ \\
External scratch $(\mathrm{cm})$ & $20.4 \pm 11.9$ & $15.4 \pm 10.1$ & $<0.001^{*}$ \\
Internal scratch $(\mathrm{cm})$ & $28.1 \pm 11.1$ & $21.7 \pm 10.3$ & $<0.001^{*}$ \\
BPI (total score) & $45.4 \pm 21.7$ & $23.7 \pm 21.2$ & $<0.001^{*}$ \\
SPADI (total score) & $61.2 \pm 24.2$ & $34.7 \pm 27.2$ & $<0.001^{*}$ \\
\hline
\end{tabular}

Values are presented as mean \pm standard deviation.

${ }^{*} \mathrm{p}<0.05$.

Table 3. Mean change of shoulder range of motion according to the arm position 2 weeks after injection

\begin{tabular}{lllcc}
\hline \multicolumn{1}{c}{ Arm position } & Abdomen & Cross & Hanging & p-value \\
\hline Abduction $\left(^{\circ}\right)$ & $23.6 \pm 19.7$ & $22.2 \pm 20.9$ & $10.0 \pm 7.8$ & 0.194 \\
\hline Flexion $\left(^{\circ}\right)$ & $14.3 \pm 18.1$ & $16.7 \pm 16.0$ & $8.5 \pm 8.2$ & 0.554 \\
\hline External rotation $\left(^{\circ}\right)$ & $18.6 \pm 10.7$ & $12.8 \pm 14.2$ & $5.0 \pm 6.2$ & 0.062 \\
\hline Internal rotation $\left(^{\circ}\right)$ & $16.0 \pm 15.5$ & $16.7 \pm 15.8$ & $11.0 \pm 6.6$ & 0.720 \\
\hline External scratch $(\mathrm{cm})$ & $-6.1 \pm 5.3$ & $-5.8 \pm 3.8$ & $-3.7 \pm 2.9$ & 0.427 \\
\hline Internal scratch $(\mathrm{cm})$ & $-6.7 \pm 2.9$ & $-6.6 \pm 6.9$ & $-5.9 \pm 5.2$ & 0.739 \\
\hline
\end{tabular}

Values are presented as mean \pm standard deviation.

Table 4. Mean change of total BPI and SPADI scores according to the arm position 2 weeks after injection

\begin{tabular}{lcclc}
\hline \multicolumn{1}{c}{ Arm position } & Abdomen & Cross & Hanging & p-value \\
\hline Total BPI & $25.1 \pm 15.7$ & $23.6 \pm 18.0$ & $11.6 \pm 6.1$ & 0.121 \\
Total SPADI & $35.0 \pm 14.2$ & $30.9 \pm 28.9$ & $16.5 \pm 10.3$ & 0.108 \\
\hline
\end{tabular}

Values are presented as mean \pm standard deviation.

BPI, Brief Pain Inventory; SPADI, Shoulder Pain and Disability Index.

\section{Comparisons of the GH joint space}

The length of the GH joint space measured by ultrasonography scans was $2.88 \pm 0.75 \mathrm{~mm}$ in abdomen position, $2.93 \pm 0.89 \mathrm{~mm}$ in cross position and $2.82 \pm 0.79 \mathrm{~mm}$ in hanging position (Fig. 3). There were no significant differences among the three arm positions as analyzed by repeated ANOVA determinations ( $\mathrm{p}=0.429$ ).

\section{Difference in the changes of efficacy measures}

When we analyzed the difference in treatment efficacy according to arm position in the shoulder ROM, for example, abduction improved $23.6^{\circ} \pm 19.7^{\circ}, 22.2^{\circ} \pm 20.9^{\circ}$, and $10.0^{\circ} \pm 7.8^{\circ}$ for abdomen, cross and hanging positions, respectively. However, there were no significant differences among the three arm positions (Table 3). Changes in

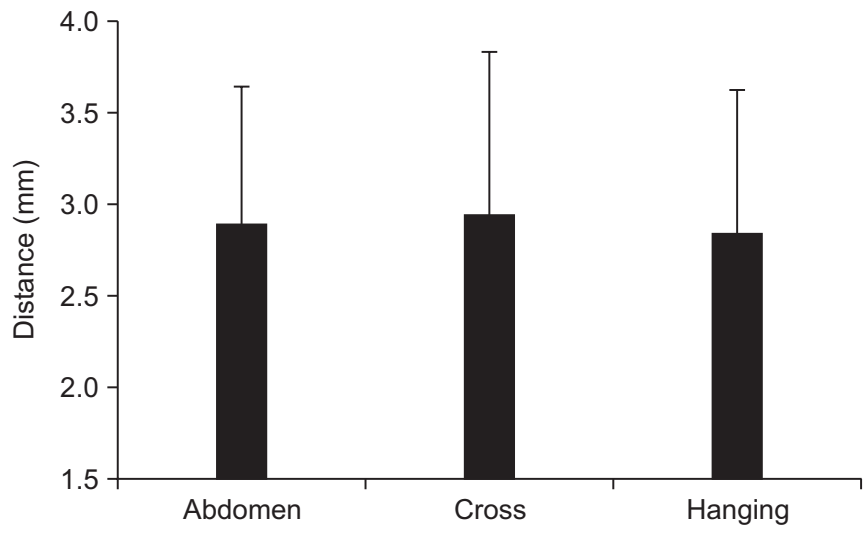

Fig. 3. The length of glenohumeral joint space in ultrasonography for each arm position. 
total BPI scores were $25.1 \pm 15.7,23.6 \pm 18.0$, and $11.6 \pm 6.1$; and total SPADI score were $35.0 \pm 14.2,30.9 \pm 28.9$, and $16.5 \pm 10.3$, respectively. There was no significant difference among the three arm positions (Table 4 ).

\section{DISCUSSION}

In this study, we found that the arm in the hanging position with internal rotation during shoulder IA-USG is a feasible position in addition to the two previously described arm positions, abdomen and cross. The hanging position provides more internal rotation and increased patients' comfort than the abdomen position. Our results indicate that the improvement in the shoulder ROM, total BPI and SPADI were not statistically different among the three positions.

However, to our surprise, the lengths of GH joint space measured using ultrasonography in the three positions were also not different. In normal anatomy, the GH joint space increases as the degree of internal rotation of the shoulder increases. However, this may not be true for patients with posterior capsule tightness. As expected, Tyler et al. [21,22] reported that posterior capsular tightness was correlated with a decrease in internal rotation. Therefore, the degree of internal rotation in patients may not be sufficient to reveal changes in the GH joint space. This may explain why we could not find any difference in the GH joint space among the three positions. The intra-articular steroid injection is commonly used to treat adhesive capsulitis of the shoulder, regardless of the underlying etiology [6]. Ultrasonography offers an accurate alternative to fluoroscopy for the injection of steroid to the shoulder joint [12]. It can be easily used in an outpatient setting without the use of ionizing radiation and iodinated contrast material. Both anterior and posterior approaches to GH joint injection have previously been reported [7-12]. The anterior approach used a vertical needle path of access to the joint. Such techniques can reduce operator confidence by preventing the continuous real-time visualization of the position of the needle-tip. Without visualizing the important anatomic landmark of the anterior glenoid labrum, there is a risk of labral damage. The posterior approach performed under real-time ultrasonography control avoids any potential for accidental puncture or injection of the major axillary neurovascular structures that may exist with the anterior approach [9].

In an outpatient setting, the most commonly used positions for the posterior approach are the abdomen and cross positions. In our study, we used the hanging position which was a hanging arm with internal rotation of the shoulder to increase the GH joint space. For patients who cannot tolerate abdomen or cross positions due to shoulder pain and a limited ROM, the hanging position is a useful alternative since all patients in our study with adhesive capsulitis showed improvement after intraarticular steroid injection in this position.

Intra-articular steroid injection under real-time ultrasonography control was performed at the first position in each patient. The changes of ROM and functional scores with the hanging position were smaller than those with the abdomen or cross positions. The degree of internal shoulder rotation at baseline was also smaller in the hanging position, although not statistically significant. This may be due to the inclusion of more severely involved patients in the hanging position, resulting in relatively small improvements after injection.

In our study, we did not confirm the success of intraarticular placement with MRI or fluoroscopy as in other studies. This was not necessary because we verified the successful location of the needle with the real-time ultrasonography image. With successful needle placement, the joint capsule was distended as shown in Fig. 4. The amount of experience with the intra-articular injection

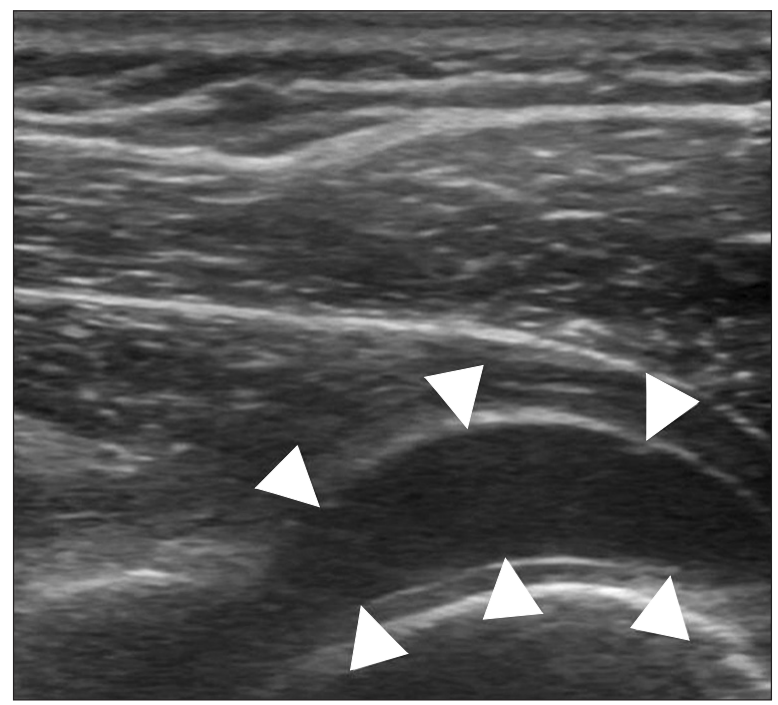

Fig. 4. The joint capsule was distended (arrowheads) after successful needle placement and injection. 
technique may affect the result obtained, although this has not been proven as yet. We did not compare the effect of experience in our study because the procedure was performed by only one physiatrist. A study which examined the accuracy of GH joint injections by personnel with different levels of experience showed that the level of experience was not a significant factor [23].

It may have been useful to differentiate the three positions in terms of patient comfort during the procedure. We tried to evaluate the level of comfort for the three positions using a visual analogue scale with a few patients during a pilot study. However, all patients stated that they felt no differences among the three positions. Thus, we decided not to include the scale for discomfort in the study. The level of discomfort may be different in extreme cases where patients with severe limitation of motion are unable to reach their hand to the opposite shoulder.

In conclusion, for most patients for whom the posterior approach with ultrasonography to treat adhesive capsulitis of shoulder is used, the hand of the affected shoulder is placed on the contralateral shoulder (cross position) or on the contralateral abdomen (abdomen position) to rotate internally the affected shoulder. However, for patients with adhesive capsulitis who cannot achieve or maintain either of those positions, an alternative treatment method may be scanning and injection with the arm in the hanging position to rotate the shoulder internally.

\section{CONFLICT OF INTEREST}

No potential conflict of interest relevant to this article was reported.

\section{REFERENCES}

1. Grey RG. The natural history of "idiopathic" frozen shoulder. J Bone Joint Surg Am 1978;60:564.

2. Neviaser JS. Adhesive capsulitis and the stiff and painful shoulder. Orthop Clin North Am 1980;11:327-31.

3. Binder AI, Bulgen DY, Hazleman BL, Roberts S. Frozen shoulder: a long-term prospective study. Ann Rheum Dis 1984;43:361-4.

4. Murnaghan JP. Adhesive capsulitis of the shoulder: current concepts and treatment. Orthopedics 1988;11: 153-8.
5. Stitik TP, Foye PM, Fossati J. Shoulder injections for osteoarthritis and other disorders. Phys Med Rehabil Clin N Am 2004;15:407-46.

6. Shah N, Lewis M. Shoulder adhesive capsulitis: systematic review of randomized trials using multiple corticosteroid injections. Br J Gen Pract 2007;57:662-7.

7. Valls R, Melloni P. Sonographic guidance of needle position for MR arthrography of the shoulder. AJR Am J Roentgenol 1997;169:845-7.

8. Vierola H. Ultrasonography-guided contrast media injection to shoulder joint using a posterior approach: a technique worth trying. Acta Radiol 2004;45:616-7.

9. Zwar RB, Read JW, Noakes JB. Sonographically guided glenohumeral joint injection. AJR Am J Roentgenol 2004;183:48-50.

10. Neethling-du Toit M, de Villiers R. Anterior approach v. posterior approach-ultrasound-guided shoulder arthrogram injection. S Afr J Radiol 2008;12:60-2.

11. Kim MW, Kim JS, Ko YJ, Lee WI, Kim JM, Yun JS. Ultrasonography guided glenohumeral injection using an anterior approach: a cadaveric study. J Korean Acad Rehabil Med 2009;33:215-8.

12. Park KD, Nam HS, Kim TK, Kang SH, Lim MH, Park Y. Comparison of sono-guided capsular distension with fluoroscopically capsular distension in adhesive capsulitis of shoulder. Ann Rehabil Med 2012;36:88-97.

13. Hannafin JA, Chiaia TA. Adhesive capsulitis. A treatment approach. Clin Orthop Relat Res 2000;(372):95109.

14. Breglio L. A system of orthopaedic medicine. J Hand Ther 1996;9:412-3.

15. Tveita EK, Ekeberg OM, Juel NG, Bautz-Holter E. Range of shoulder motion in patients with adhesive capsulitis; intra-tester reproducibility is acceptable for group comparisons. BMC Musculoskelet Disord 2008;9:49.

16. Tveita EK, Tariq R, Sesseng S, Juel NG, Bautz-Holter E. Hydrodilatation, corticosteroids and adhesive capsulitis: a randomized controlled trial. BMC Musculoskelet Disord 2008;9:53.

17. Arslan S, Celiker R. Comparison of the efficacy of local corticosteroid injection and physical therapy for the treatment of adhesive capsulitis. Rheumatol Int 2001; 21:20-3.

18. Yun YH, Mendoza TR, Heo DS, Yoo T, Heo BY, Park HA, et al. Development of a cancer pain assessment tool in Korea: a validation study of a Korean version of 
the brief pain inventory. Oncology 2004;66:439-44.

19. Roach KE, Budiman-Mak E, Songsiridej N, Lertratanakul Y. Development of a shoulder pain and disability index. Arthritis Care Res 1991;4:143-9.

20. Woodward TW, Best TM. The painful shoulder: part I. Clinical evaluation. Am Fam Physician 2000;61:307988.

21. Tyler TF, Roy T, Nicholas SJ, Gleim GW. Reliability and validity of a new method of measuring posterior shoulder tightness. J Orthop Sports Phys Ther 1999;29:262-9; discussion 270-4.

22. Tyler TF, Nicholas SJ, Roy T, Gleim GW. Quantification of posterior capsule tightness and motion loss in patients with shoulder impingement. Am J Sports Med 2000;28:668-73.

23. Tobola A, Cook C, Cassas KJ, Hawkins RJ, Wienke JR, Tolan S, et al. Accuracy of glenohumeral joint injections: comparing approach and experience of provider. J Shoulder Elbow Surg 2011;20:1147-54. 